\title{
Dry Surface Micromanipulation Using an Untethered Magnetic Microrobot
}

\author{
Omid Youssefi \\ Department of Mechanical and Industrial Engineering \\ University of Toronto \\ Toronto, ON \\ omidy@mie.utoronto.ca
}

\author{
Eric Diller \\ Department of Mechanical and Industrial Engineering \\ University of Toronto \\ Toronto, ON \\ ediller@mie.utoronto.ca
}

\begin{abstract}
Precise micromanipulation tasks are typically performed using micromanipulators that require an accessible workspace to reach components. However, many applications have inaccessible or require sealed workspaces. This paper presents a novel magnetically-guided, and untethered, actuation method for precise and accurate positioning of microcomponents on dry surface within a remote workspace using a magnetic microrobot. By use of an oscillatory and uniform magnetic field, the magnetic microrobot can traverse on a dry surface with fine step size and accurate open-loop vector following, $3 \%$ and $2 \%$ of its body-length, respectively (step size of $7 \mu \mathrm{m}$ ). While maintaining precise positioning capability, the microrobot can manipulate and carry other microcomponents on the dry surface using direct pushing or grasping using various attachments, respectively. We demonstrate and characterize the untethered micromanipulation capabilities of this method using a $3 \mathrm{~mm}$ cubic microrobot for use in applications such as microassembly and cargo transport.
\end{abstract}

Keywords: Microrobotics; Microfactory; Microassembly; Magnetic Micromanipulation; Untethered Actuation.

\section{INTRODUCTION}

Industrial robotic systems, such as robotic arms, part positioners, and sorters, have enabled rapid and precise manufacturing of large-scale assemblies. Extension of such systems into centimeter-scale manufacturing, such as in part pick-and-place for printed circuit board assembly, has also proven to be practical. However, significant challenges exist for manufacturing at the microscale level (characteristic dimensions of less than $10 \mathrm{~mm}$ ). Flexure-based mechanisms, such as the delta [1] and MEMS-based [2] positioners, can position microcomponents within a few micrometer and nanometer accuracies, respectively. High speeds and precise operation make tethered and traditional manufacturing methods suitable for widespread use. Tethered systems require an entry area for access to the workspace, which may not be possible in some applications requiring sealing. Tethered assembly of microcomponents, such as the $200 \mu \mathrm{m}$ optical prism used in [3], require a cleanroom environment. On the other hand, untethered microassembly can be performed within a smaller and sealed workspace, which is a cost-effective approach as the workspace can meet the requirements of a clean environment.

Due to the small scale of untethered microrobots, onboard power and actuation is impractical and thus, most designs in the literature rely on external actuation such as using magnetic fields [4], [5], and [6]. Most in-liquid micromanipulation methods use magnetic gradient pulling to perform micromanipulation tasks [7], [8], and [9]. In-liquid pick-and-place operations, using bubbles [10] and a magnetic microgripper [7], have proven effective in performing autonomous tasks and are well studied. However, micromanipulation on dry surface is required for many applications and it is challenging due to the strong friction and adhesion present on the small scale [5]. To avoid the stiction phenomenon altogether, levitated micromanipulation techniques, using diamagnetic [11] and acoustic [12] levitations, are used. However, to overcome the stiction forces on the surface, methods such as the stick-slip [13] and tumbling motions [14], use varying magnetic fields to induce torque instead of direct gradient pulling. Magnetic torque enables lifting and rolling of the magnetic microcomponents which indirectly result in step-wise motion on a surface.

Dry surface micromanipulation methods in the literature either have coarse stride step sizes (at least a body-length) [14], [15], and [16] that are imprecise or are uncontrolled during some portion of their stride cycle [13]. In addition, a pushing force cannot be applied using these for direct micromanipulation. Accuracy, precision, and speed are the core requirements for applications involving dry surface microassembly, where assembly force, as well as precise and efficient positioning and orienting are necessary. Most methods require complex actuation and control schemes and thus, become sensitive to external disturbances. Use of closed-loop controllers can alleviate the positioning inaccuracies, however; ability of applying pushing force is insufficient or lacking. Dry surface micromanipulation tasks, in inaccessible workspaces, require untethered, precise, and accurate locomotion with strong force application, which have not been achieved so far.

In this work, we introduce a novel method for precise positioning of a magnetic microrobot on dry surface. Unlike 
previous work, which aim to overcome the surface stiction forces, the new method of walking takes advantage of friction forces to apply strong pushing forces required for microassembly. The open-loop operation of this walking method provides accurate path following (within $2 \%$ of body-length) and has walking step sizes as small as $3 \%$ of the microrobots largest dimension, which enable precision micromanipulation and handling of various microcomponents.

\section{CONCEPT}

In this section, we introduce the new principle behind the actuation, the stepwise, and precise locomotion of a cubic magnetic microrobot on a dry surface. By taking advantage of high surface friction force, the microrobot can manipulate other microcomponents either by carrying or direct pushing.

\section{A. Method of Walking}

Walking is achieved via remote magnetic actuation which is a uniform and oscillatory field. A torque, $\boldsymbol{\tau}_{\mathrm{B}}$, is induced on the magnetic microrobot resulting in change of its kinematic state. As shown in Fig. 1(a), the external magnetic field vector, $\mathbf{B}$, rotates at a frequency, $\mathrm{f}$, about an axis defined by angles $\theta$ and $\beta$ which are the heading and the tilt angles, respectively. This field profile cone, as shown in Fig. 1(a), has an opening angle of $\phi$ degrees. The microrobot, with its volume magnetization vector, $\mathbf{M}$, follows this field orientation because of the induced torque, which is defined by

$$
\mathbf{\tau}_{\mathrm{B}}=\mathbf{M} \times \mathbf{B} \text {. }
$$

This two-degree-of-freedom (2-DoF) actuation, along with the dynamic interaction of the microrobot with the dry surface, result in a stepwise motion with a full-cycle stepping shown in Fig. 1(a). The motion characteristics (i.e. step precision, speed, and path accuracy) are finely tunable by varying the field parameters. To achieve a consistent stepping motion, a suitable pose must be established such that the microrobot contacts the surface with exactly two defined points. This is the case for most flat-edged contacts where the line contact can be approximated with two points dictated by surface roughness profile. However, for accurate and repeatable motion, the two points can be physically fabricated to be more prominent to ensure repeatable contact over successive stepping cycles and during micromanipulation tasks such as pushing. In this work, an attachment, with two sharp corners, is glued underneath the microrobot for use in the experiments. Unlike tumbling and rolling methods for locomotion of flat-edged geometries, [14] and [15], the point contact reduces the contact friction, however; it enables pushing of microcomponents with precision using sharp-point anchorage, controllable steering, and precise positioning.

\section{B. Method of Force Application}

Two significant parameters of interaction between the microrobot and the surface, especially at the microscale level, are: the friction, $\mathbf{F}_{\mathbf{N}}$; and the surface adhesion, $\mathbf{F}_{\mathbf{a}}$, forces. These correlated forces enable the microrobot to use the
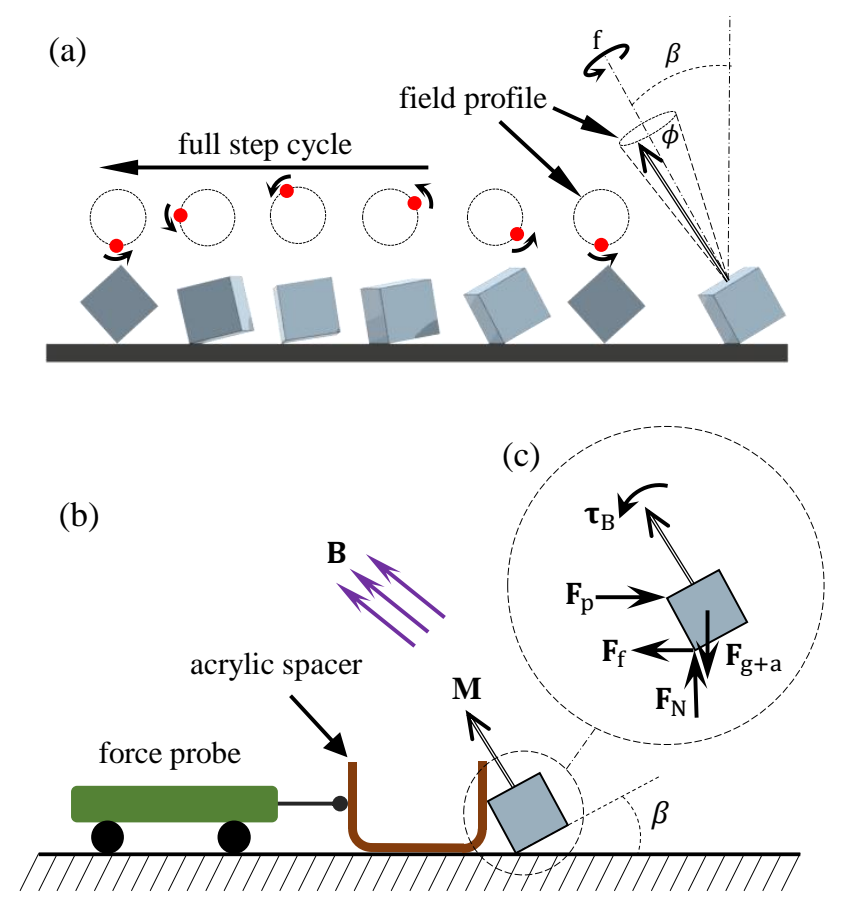

Figure 1. Conceptual schematic of walking and pushing tasks: (a) walking motion sequence during a full step cycle; (b) schematic of the force measurement setup; and (c) free-body diagram showing the forces and torque induced on the block at an instant of a pushing task.

friction force, $\mathbf{F}_{\mathbf{f}}$, to apply an equal and opposite force, $\mathbf{F}_{\mathbf{p}}$, to other microcomponents. A free-body diagram showing forces during a pushing task (Fig. 1(b)) is shown in Fig. 1(c). By adjusting the heading angle, the microrobot can push microcomponents around on a surface on a specific path, or towards a goal pose in a semi-holonomic fashion.

\section{MODELLING AND CHARACTERIZATION}

In this section, we present the magnetic field actuation scheme, and show the characteristics of a pushing task.

\section{A. Time Varying Magnetic Field}

A uniform magnetic field (field with no gradient) is necessary for the 2-DoF actuation of the microrobot. The smooth rotation of this field along the circular profile shown in Fig. 1(a), is necessary to prevent jerks, which would result in undesired movement or jumping of the microrobot. Thus, a central controller, with accurate time keeping capability, is essential to ensure the smooth actuation. The magnetic field is defined by

$$
\mathbf{B}=\mathrm{B}\left(\mathrm{R}_{\mathrm{z}}(\theta) \mathrm{R}_{\mathrm{y}^{\prime}}(\beta)\right)\left[\begin{array}{c}
\sin (\phi / 2) \cos (2 \pi \mathrm{ft}) \\
\sin (\phi / 2) \sin (2 \pi \mathrm{ft}) \\
\cos (\phi / 2)
\end{array}\right]
$$

where $\mathrm{t}$ is time, $\mathrm{B}$ is the magnitude of the magnetic flux density, $R_{z}$ is the rotation matrix about the $Z$ axis for the world frame, and $\mathrm{R}_{\mathrm{y}^{\prime}}$ is the rotation matrix about the $\mathrm{Y}$ axis for the new rotated frame. From (1), given an object with a fixed magnetization, a strong magnitude field is necessary for full rolling motion because a higher magnetic torque is 


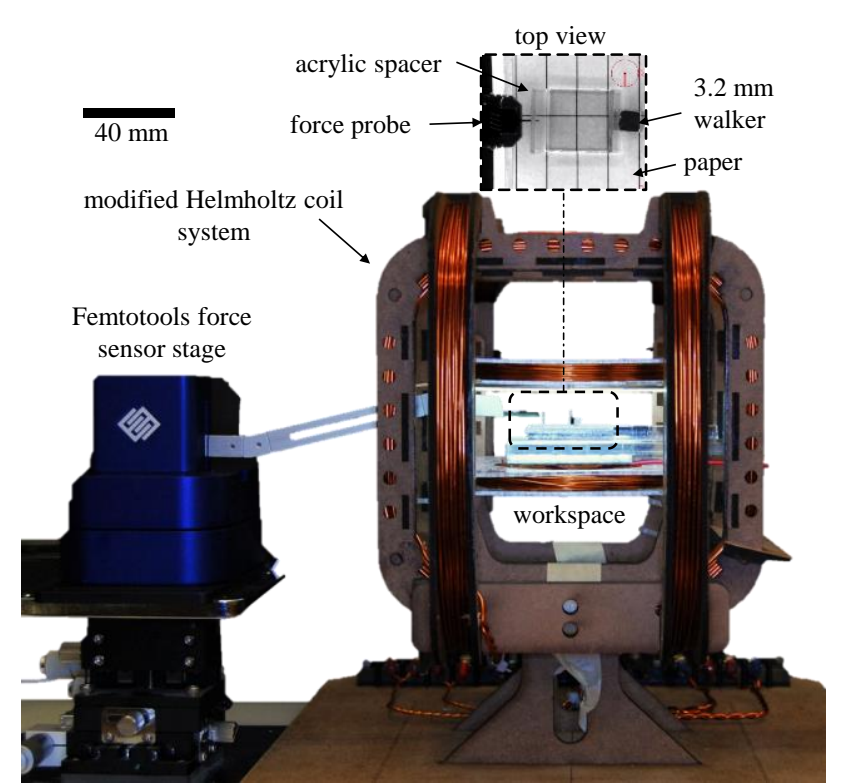

Figure 2. Experiment setup: the microrobot, the acrylic spacer, and the force sensor are placed on a paper surface within the workspace which is in the middle of custom-designed 3-axis Helmholtz coil system.

required to overcome the torque due to the weight and the surface adhesion forces from a lay-flat pose. However, with this method, since the microrobot needs to follow only the direction of the varying field with a short span, lowermagnitude field can be used. This becomes apparent for the case when a cubic microrobot is used and when $\beta=45^{\circ}$ where the magnetic torque is mostly overcoming the inertia (minimal at the microscale level and low frequency) as the weight is balanced by $\mathbf{F}_{\mathbf{N}}$. Therefore, unlike positioning achieved by rolling, a lower magnitude magnetic field can be used in certain configurations.

\section{B. Pushing Force Characterization}

Using the same magnetic field actuation in (2), the microrobot can exert a pushing force on microcomponents with a flat and vertical surface. The friction and adhesion forces anchor the contact point of the microrobot to the surface and thus, an equal and opposite force can be applied to the microcomponent. This force is applied during a portion of a full-step cycle corresponding to the configuration shown in Fig. 1(b), where the pushing force $\mathbf{F}_{\mathbf{p}}$ is achieved because of the magnetic torque $\tau_{\mathrm{B}}$. As the microrobot relies on the point of contact to apply a force, the magnitude of the force is limited by the friction force between the microrobot and the surface. A pushing experiment was performed, and the results are presented in section $\mathrm{IV}(\mathrm{B})$.

\section{RESUlTS AND DiscUSSION}

\section{A. Experimental setup - Magnetic Field and Force Sensing}

A custom-designed setup was fabricated to actuate, sense, and measure the performance of the microrobot. The prototype coil system is comprised of three pairs of nested and mutually-orthogonal square Helmholtz coils which can generate a nearly uniform magnetic field of up to $3 \mathrm{mT}$ in a workspace with dimensions of $30 \times 40 \times 40 \mathrm{~mm}$. The

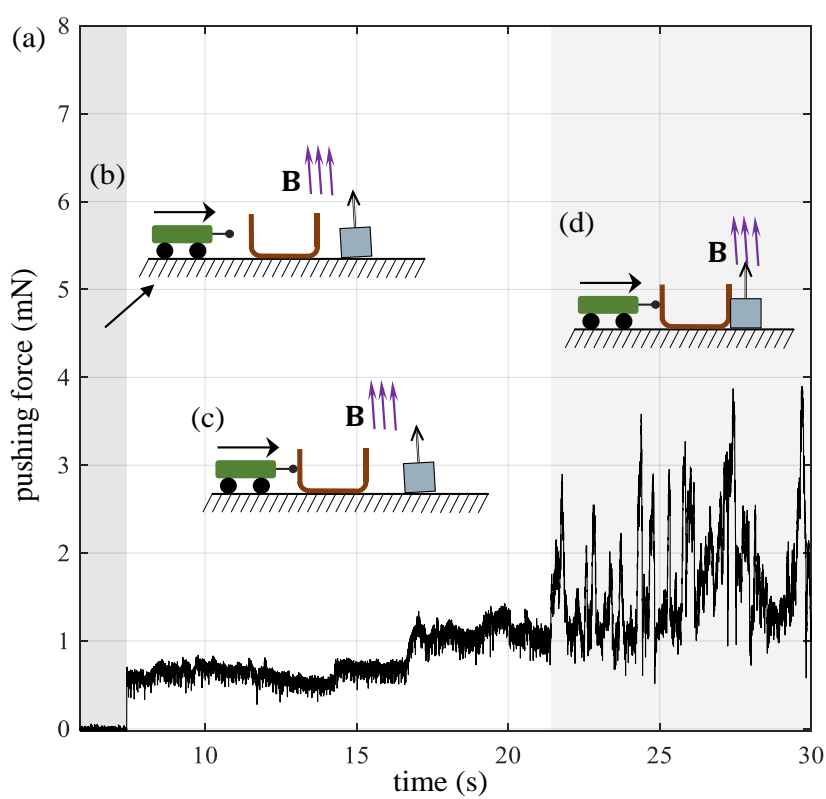

Figure 3. Friction force measurement between the microrobot and the paper surface, measured by a moving force sensor: (a) pushing force raw data; (b) interval of no contact, force sensor approaching the spacer; (c) interval of force sensor contact with the spacer; and (d) interval of microrobot contact with the spacer.

magnitude of the field is directly proportional to the current flowing in the coils which are controlled by six amplifiers (Advanced Motion Controls, 30A8) and commanded by a digital-to-analog converter card (Sensoray, Model s826). The commands are sent from a custom C-code executed on a Linux-based PC. A top-view camera (FOculus, FO123TB) is used to record position information of the microrobot. The proof-of-concept microrobot is composed of a cubic N52 magnet with side length of $3.22 \mathrm{~mm}$. A two-point attachment is glued on the bottom surface of the magnet to ensure consistent contact with the surface. To measure the pushing and friction forces, a microforce sensing probe (Femtotools FT S10000) was mounted on a moving stage with a constant feed rate.

\section{B. Pushing Friction Test Results}

To test the pushing capability of the microrobot, it was necessary to first determine the friction force present between the microrobot and the surface. This friction force was measured indirectly by using the acrylic spacer placed in between the force probe and the microrobot. The acrylic spacer provided two vertical surfaces for accurate transfer of force. Using a $3 \mathrm{mT}$ constant magnetic field, the microrobot was tilted $5^{\circ}$ towards the acrylic spacer as shown in Fig. 3(b). The force probe is moving to the right with a constant federate. During the initial approach (Fig. 3(b)), a small amount of noise is observed. Upon reaching the acrylic spacer, the sensor measured an averaged friction force of about $0.5 \mathrm{mN}$ which is the friction of the acrylic spacer with the surface. At around 17 seconds, the acrylic spacer contacted the top edge of the microrobot which corresponds to the increase in force value at that time. After the microrobot was rotated to a flat pose by the acrylic spacer, the magnetic field was turned off and the microrobot was 
pushed on the surface by the force probe (Fig. 3(c)). An average force of $1.70 \mathrm{mN}$ was measured during this interval. By subtracting the acrylic friction force, the average friction force of the microrobot was calculated to be $1.2 \mathrm{mN}$.

The highly varying friction force profile, as shown in Fig. $3(\mathrm{c})$, is due to the stick-slip characteristic of friction on dry surfaces. Thus, unlike sliding by gradient pull, a step-wise motion is preferred on dry surface. Further, the high impulse and oscillatory force application of the microrobot on other microcomponents enables the reliable micromanipulation.

\section{Open-loop Dead Reckoning Experiment and Locomotion Characterization}

To characterize the motion, the microrobot was placed on a piece of copy paper inside the coil system and the position of the microrobot was tracked over time using vision analysis post-experiment. The tilt angle, opening angle, and frequency parameters were varied to characterize the effects of each on the walking accuracy and speed. The result of the first experiment, with $\beta=20^{\circ}, \phi=8^{\circ}$, and $\mathrm{f}=1 \mathrm{~Hz}$, is shown in Fig. 4(a). A heading angle of $0^{\circ}$ (travelling to east) was set and the experiment was conducted for a duration of $34.8 \mathrm{~s}$, effectively corresponding to about 35 full stepping cycles. A distance of $24.81 \mathrm{~mm}$ was covered during this duration in which, the effective per-step resolution is $0.7 \mathrm{~mm}(22 \%$ of body-length). The on-course path deviation error was characterized by RMSE, as shown as an inset in Fig. 4, which is $0.059 \mathrm{~mm}$ ( $1.8 \%$ of body-length). The end position of the microrobot was too close to the expected position for characterizing the offset using vision. Despite an open-loop actuation, the vector following is precise and accurate. This is as a result of the oscillatory and fine stepping of the microrobot on the dry surface. A separate experiment, using $0.25 \mathrm{~mm}$ cubic magnet on glass, was performed with step size and path deviation of $2 \%$ and $3 \%$ of body-length, respectively.

To characterize the speed, three opening angles $\left(\phi=8^{\circ}\right.$, $10^{\circ}$, and $15^{\circ}$ ) were selected and a magnetic field of $3 \mathrm{mT}$ was applied. The speed depends on all field parameters, however; the most notable dependability is on the frequency and the opening angle. The distance of the CoM was measured using post-experiment vision analysis and the results are shown in Fig. 4(b). Linear fits were calculated, and the average speeds were found to be $0.714,1.004$, and $1.542 \mathrm{~mm} / \mathrm{s}$ for $\phi=8^{\circ}$, $10^{\circ}$, and $15^{\circ}$, respectively. These sub-body length speeds and walking steps are finely adjustable making them suitable for precision positioning and locomotion. Using this microrobot, a maximum linear speed of $4.23 \mathrm{~mm} / \mathrm{s}$ (131\% of body length) was achieved with $\phi=45^{\circ}, \beta=30^{\circ}$, and $\mathrm{f}=1 \mathrm{~Hz}$. The speed can also be increased by increasing the frequency. However, at high frequencies, a higher magnitude magnetic field is required because of the inertia of the microrobot. The distance plot, shown in Fig. 4(b), shows the reliability of open-loop actuation on a uniform surface. Presence of external disturbances will affect the performance, however; it can be compensated by using active feedback.
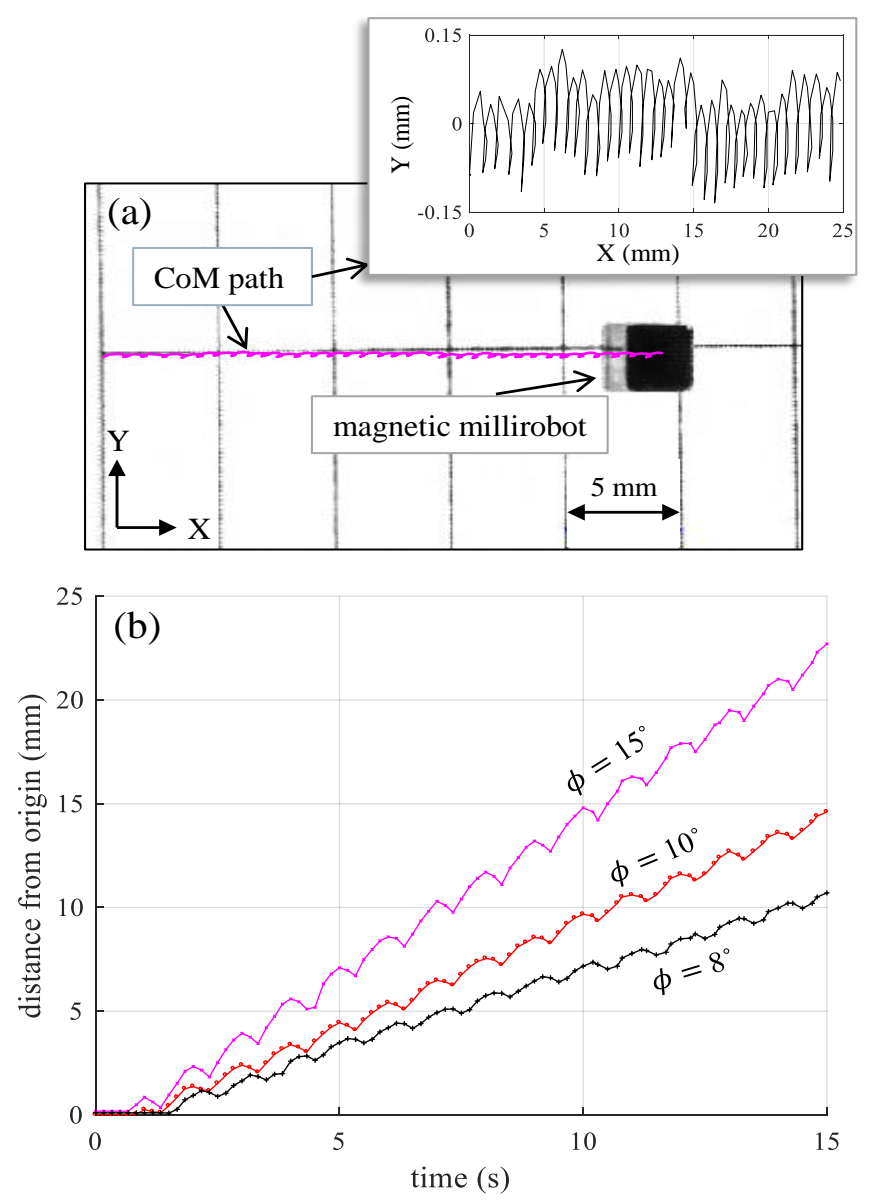

Figure 4. Precision open-loop walking characteristics of the magnetic walker: (a) top view of the microrobot at the end position with the walking path traced, $\beta=20^{\circ}, \phi=8^{\circ}, \mathrm{f}=1 \mathrm{~Hz}$; and (b) plot of distance of center of mass from the starting location for span angles, $\phi=8^{\circ}, 10^{\circ}$, and $15^{\circ}$ where $\mathrm{f}$ $=1 \mathrm{~Hz}$.

\section{CONCLUSION AND FUTURE WORK}

The new micromanipulation method presented in this paper allows for simple yet, precise open-loop and untethered positioning and micromanipulation of various microcomponents on a dry surface actuated by an external magnetic field. Unlike previous work, the presented microrobot can push other components while maintaining a reasonable positioning accuracy, and it can accurately traverse on a dry surface with different stepping size and speeds, which are finely tunable. In-place change of heading is possible by using some tilt angle giving this microrobot a holonomic characteristic to its otherwise nonholonomic motion. Due to the precise nature of the locomotion, highspeed travel is limited to 1-2 body lengths per second. For high speeds, rolling motion can be used by trading off stepping resolution and precision pushing capability. In addition, a reasonably flat surface is required for repeatable and open-loop path following, however; significant potential exists for integrating a closed-loop controller for making this method suitable for use in many industrial applications such as microassembly and cargo transport at the milli- and microscale levels. 


\section{REFERENCES}

[1] H. McClintock, F. Temel, N. Doshi, J. Koh and R. Wood, "The milliDelta: A high-bandwidth, high-precision, millimeter-scale Delta robot", Science Robotics, vol. 3, no. 14, p. eaar3018, 2018.

[2] H. Chen, D. Jiang and I. Hagiwara, "Development of Novel XY Micropositioning Stage", Key Engineering Materials, vol. 407-409, pp. 103-106, 2009.

[3] B. Li et al., "Hybrid Intravascular Ultrasound and Optical Coherence Tomography Catheter for Imaging of Coronary Atherosclerosis," Catheterization and Cardiovascular Interventions, vol. 81, pp. 494507, 2013.

[4] Kummer, Michael P., et al. "OctoMag: An electromagnetic system for 5-DOF wireless micromanipulation." IEEE Transactions on Robotics 26.6 (2010): 1006-1017.

[5] E. Diller and M. Sitti, "Micro-Scale Mobile Robotics," Foundations and Trends in Robotics, vol. 2, no. 3, pp. 143-259, 2013.

[6] E. Diller, J. Giltinan, G. Lum, Z. Ye, and M. Sitti, "Six-degree-offreedom magnetic actuation for wireless microrobotics," International Journal of Robotics Research, vol. 35, no. 1, pp. 114-128, 2016.

[7] J. Zhang, O. Onaizah, K. Middleton, L. You, and E. Diller, "Reliable Grasping of Three-Dimensional Untethered Mobile Magnetic Microgripper for Autonomous Pick-and-Place", IEEE Robotics and Automation Letters, vol. 2, issue 2, pp. 835-840, 2017.

[8] Pieters, Roel, et al. "RodBot: A rolling microrobot for micromanipulation." Robotics and Automation (ICRA), 2015 IEEE International Conference on. IEEE, 2015.

[9] Fluid-based micromanipulation: Z. Ye, E. Diller and M. Sitti, "MicroManipulation Using Rotational Fluid Flows Induced by Wireless Magnetic Micro-Manipulators," Journal of Applied Physics, vol. 112, no. 6, 064912, 2012.

[10] J. Giltinan, E. Diller, and M. Sitti, "Programmable Assembly of Heterogeneous Microparts by an Untethered Mobile Capillary Microgripper," Lab on a Chip, vol. 16, pp. 4445-4457, 2016.

[11] Hsu, Allen, et al. "Automated 2D micro-assembly using diamagnetically levitated milli-robots." Manipulation, Automation and Robotics at Small Scales (MARSS), 2017 International Conference on. IEEE, 2017.

[12] D. Foresti, M. Nabavi, M. Klingauf, A. Ferrari and D. Poulikakos, "Acoustophoretic contactless transport and handling of matter in air", Proceedings of the National Academy of Sciences, vol. 110, no. 31, pp. 12549-12554, 2013.

[13] Pawashe, Chytra, Steven Floyd, and Metin Sitti. "Modeling and experimental characterization of an untethered magnetic micro-robot." The International Journal of Robotics Research 28.8 (2009): 10771094.

[14] Jing, Wuming, Nicholas Pagano, and David J. Cappelleri. "A novel micro-scale magnetic tumbling microrobot." Journal of Micro-Bio Robotics 8.1 (2013): 1-12.

[15] C. Bi, M. Guix, B. Johnson, W. Jing and D. Cappelleri, "Design of Microscale Magnetic Tumbling Robots for Locomotion in Multiple Environments and Complex Terrains", Micromachines, vol. 9, no. 2, p. $68,2018$.

[16] S. Jeon, "A sphericon-shaped magnetic microrobot rolling on a surface actuated by an external wobbling magnetic field", AIP Advances, vol. 7, no. 5, p. 056708, 2017. 